\title{
CONCORDANCE AND ISOTOPY OF PL EMBEDDINGS
}

\author{
J. F. P. HUDSON
}

Communicated by J. Milnor, November 18, 1965

Concordance and isotopy give two equivalence relations between embeddings of manifolds in manifolds. The results announced in this paper show that, for PL embeddings of a compact manifold with codimension $\geqq 3$, these two relations are the same.

One effect of this is that unknotting theorems may be deduced immediately from embedding theorems. Such an unknotting theorem is given here (Theorem 4).

Definitions. Let $M$ and $Q$ be PL manifolds of dimensions $m$ and $q$ respectively. Let $I$ denote the closed unit interval of the real line. A concordance of $M$ in $Q$ is a PL embedding $h: M \times I \rightarrow Q \times I$ such that $h(M \times 0) \subset(Q \times 0)$ and $h(M \times 1) \subset Q \times 1$.

An isotopy of $M$ in $Q$ is a concordance which is level-preserving, that is to say, $h(M \times t) \subset Q \times t$ for all $t$ in $I$. $h_{t}: M \rightarrow Q$ is used to denote the embedding defined by $h(x, t)=\left(h_{t} x, t\right)$ for all $x$ in $M$.

This notation is also used with $t=0$ or 1 , when $h$ is any concordance.

If $X \subset M$, a concordance (or isotopy) $h$ of $M$ in $Q$ is fixed on $X$ if $h(x, t)=\left(h_{0} x, t\right)$ for all $(x, t)$ in $X \times I$.

Now suppose that $M$ and $Q$ have boundaries $\partial M$ and $\partial Q$ respectively, possibly empty.

An embedding $i: M \rightarrow Q$ is allowable if $i^{-1} \partial Q$ is an $(m-1)$-submanifold of $\partial M$, possibly empty or the whole of $\partial M$.

A concordance (or isotopy) $h$ of $M$ in $Q$ is allowable if $h^{-1}(Q \times 0)$ $=M \times 0, h^{-1}(Q \times 1)=M \times 1, h_{0}$ is an allowable embedding, and $h^{-1}(\partial Q \times I)=\left(h_{0}^{-1} \partial Q\right) \times I$.

Two embeddings $f$ and $g$ of $M$ in $Q$ are concordant if there is a concordance $h$ of $M$ in $Q$ with $h_{0}=f$ and $h_{1}=g$.

Similarly we talk of two embeddings being isotopic, allowably concordant, concordant keeping a subset of $M$ fixed, etc.

An ambient isotopy of $Q$ is an isotopy of $Q$ in itself which is a PL homomorphism and with $h_{0}$ equal to the identity.

Two embeddings $f$ and $g$ of $M$ in $Q$ are ambient isotopic if there is an ambient isotopy $h$ of $Q$ such that $h_{1} f=g$.

By the isotopy extension theorem [1] and [2], the conditions allowably isotopic and ambient isotopic are equivalent for embeddings of compact manifolds with codemension $\geqq 3$.

TheOREM 1. Let $h: M \times I \rightarrow Q \times I$ be an allowable concordance. If $M$ 
is compact and $q-m \geqq 3$, then there is an ambient isotopy $H$ of $Q \times I$, fixed on $(Q \times 0) \cup(Q \times 1)$, such that $H_{1} h$ is an isotopy.

TheOREM 2. If $h: M \times I \rightarrow Q \times I$ is an allowable concordance whose restriction to $h_{0}^{-1} \partial Q \times I$ is level-preserving, and if $M$ is compact and $q-m$ is $\geqq 3$, then there is an ambient isotopy $H$ of $Q \times I$, fixed on $\partial(Q \times I)$, such that $H_{1} h$ is an isotopy.

Theorem 3. Let $h: M \times I \rightarrow Q \times I$ be an allowable concordance whose restriction to $(\partial M) \times I$ is level-preserving. If $M$ is compact and $q-m \geqq 3$, then there is an ambient isotopy $H$ of $Q \times I$, fixed on $\partial(Q \times I)$ $\cup\left[\left(h_{0} \partial M\right) \times I\right]$, such that $H_{1} h$ is an isotopy.

The isotopy extension theorem, [1] and [2], now gives the corollaries:

If $f$ and $g$ are embeddings of $M$ in $Q$, where $M$ is compact and $q-m \geqq 3$, then

(i) if $f$ and $g$ are allowably concordant, they are ambient isotopic,

(ii) if $f$ and $g$ are allowably concordant keeping $f^{-1} \partial Q$ fxed, then they are ambient isotopic keeping $\partial Q$ fixed,

(iii) if $f$ and $g$ are allowably concordant keeping $\partial M$ fixed, then they are ambient isotopic keeping $\partial Q \cup f(\partial M)$ fixed.

Applying Irwin's embedding theorem to $M \times I$ and $Q \times I$ and then applying corollary (iii) gives:

Theorem 4. Let $f, g: M \rightarrow Q$ be allowable $\mathrm{PL}$ embeddings which are homotopic keeping $\partial M$ fixed. If $M$ is compact, $q-m \geqq 3, M$ is $(2 m-q+1)$-connected, $Q$ is $(2 m-q+2)$-connected and $\partial M-f^{-1}(\partial Q)$ is either empty or $(2 m-q)$-connected, then $f$ and $g$ are ambient isotopic keeping $\partial Q$ and $f(\partial M)$ fixed.

\section{BIBLIOGRAPHY}

1. J. F. P. Hudson and E. C. Zeeman, On combinatorial isotopy, Inst. Hautes Etudes Sci. Publ. No. 19 (1964), 69-94.

2. J. F. P. Hudson, Extending PL isotopies, Proc. London Math. Soc. (to appear).

3. M. C. Irwin, Embeddings of polyhedral manifolds, Ann. of Math. (2) 82 (1965), $1-14$.

4. E. C. Zeeman, Isotopies of manifolds, Topology of 3-manifolds, (ed., M. K. Fort), Prentice-Hall, Englewood Cliffs, N. J., 1962.

CAmbridge University, England 